\title{
TENACIDADE DA MADEIRA COMO FUNÇÃO DA DENSIDADE APARENTE ${ }^{1}$
}

\author{
Diego Henrique de Almeida ${ }^{2}$, Ricardo de Mello Scaliante², André Luis Christoforo ${ }^{3}$, Luciano Donizeti \\ Varanda ${ }^{4}$, Francisco Antonio Rocco Lahr ${ }^{5}$, Antonio Alves Dias ${ }^{5}$ e Carlito Calil Junior ${ }^{5}$
}

\begin{abstract}
RESUMO - O conhecimento do comportamento da madeira mediante ações de curta duração contribui para um projeto estrutural mais seguro, entretanto não sendo a tenacidade parte integrante das propriedades mecânicas comumente investigadas na caracterização deste material. Este trabalho objetivou avaliar, com o uso de modelos de regressões polinomial linear, quadrática e cúbica, a possibilidade da estimativa da tenacidade com o conhecimento da densidade aparente de seis espécies de madeira (Angico, Eucalipto, Jatobá, Paricá, Pinus e Teca). Os resultados dos modelos de regressão evidenciaram a significância e representatividade de todos os ajustes investigados, consistindo o polinomial cúbico o mais eficiente na estimativa da tenacidade da madeira.
\end{abstract}

Palavras-chave: Tenacidade; Modelos de regressão; Densidade aparente.

\section{WOODEN TOUGHNESS AS FUNCTION OF THE APPARENT DENSITY}

\begin{abstract}
The knowledge on the behavior of wood in situations of short load contributes to the development of a safer structural design. However, toughness is not part of the mechanical properties commonly investigated in the characterization of this material. Therefore, the present study aimed to evaluate, the possibility to estimate the toughness with the knowledge of the apparent density of 6 Brazilian tropical wooden species (Angico, Eucalyptus, Jatobá, Paricá, Pine and Teak), by using linear, quadratic and cubic polynomial regression models. The results highlighted the significance and representation of all adjustments investigated, and the cubic polynomial was the most efficient for estimating the wood toughness.
\end{abstract}

Keywords: Toughness; Regression models; Apparent density.

\section{INTRODUÇÃO}

A utilização da madeira nas mais diversas finalidades depende do conhecimento de suas propriedades (físicas, químicas, mecânicas e anatômicas) para utilização racional desse material que, por ser proveniente de fontes naturais, preenche perfeitamente os requisitos impostos pelo atual apelo ambiental de produtos e serviços fornecidos pelo homem.

Uma das propriedades mecânicas dos materiais é a tenacidade. Segundo Callister Junior (1999), a tenacidade é a capacidade de certo material absorver energia num pequeno intervalo de tempo. Calil Junior et al. (2003) dissertaram que a madeira é um material tenaz, quando comparado a outros materiais muito utilizados atualmente, a exemplo do aço e do concreto.

Por ser material tenaz, a madeira é indicada para utilizações em pontes, dormentes de ferrovias, artigos esportivos, cabos de ferramentas, mourões de currais e em outras situações onde existem impactos ou cargas cíclicas (RITTER, 1990; CALIL JUNIOR et al., 2006; HAMM et al., 2007; FREITAS, 2009).

O conhecimento da tenacidade permite estimar deteriorações ou anomalias em peças que estejam sendo utilizadas em estruturas de madeira. A degradação da

\footnotetext{
${ }^{1}$ Recebido em 11.06.2013 aceito para publicação em 03.12.2013.

${ }^{2}$ Programa de Pós-Graduação em Engenharia de Estruturas (LaMEM/SET/EESC/USP). E-mail: <almeidadh@usp.br> e <scaliante@usp.br>.

${ }^{3}$ Departamento de Engenharia Civil, Universidade Federal de São Carlos (DECiv/ UFSCar). E-mail: <alchristoforo@yahoo.com.br>.

${ }^{4}$ Programa de Pós-Graduação em Engenharia de Materiais (LaMEM/SMM/EESC/USP). E-mail: <lu.varanda@hotmail.com>.

${ }^{5}$ Laboratório de Madeiras e de Estruturas de Madeira, Departamento de Engenharia de Estruturas, Escola de Engenharia de São Carlos, Universidade de São Paulo(LaMEM/SET/EESC/USP). E-mail: <frocco@sc.usp.br>, <dias@sc.usp.br>e <calil@sc.usp.br> .
} 
madeira por agentes xilófagos (fungos, bactérias e insetos) pode diminuir em até $50 \%$ a tenacidade da madeira utilizada em estruturas (STOLF, 2000; RIDOUT, 2009; ABRUZZI et al., 2012).

Moreschi (2005), Lahr (1990), Stolf (2000) eAlmeida et al. (2011) citaram alguns fatores que influenciam a tenacidade, como a forma e as dimensões dos corpos de prova, o teor de umidade, as propriedades anatômicas da madeira, a angulação das fibras, a densidade da madeira e a temperatura da madeira no momento do ensaio.

A tenacidade tem sido usada para descrever características da madeira, estando relacionada com outras propriedades, como as resistências ao cisalhamento e a tração normal às fibras. Entretanto, a tenacidade não deve ser considerada separadamente para caracterizar uma espécie, sendo necessária a avaliação conjunta dos resultados de outros ensaios (ASKELAND, 1994).

Entre outras propriedades importantes da madeira, pode-se citar a densidade aparente, que é a relação entre a sua massa e o seu volume na umidade de equilíbrio. A partir da densidade aparente, é possível estimar outras propriedades de resistência e de rigidez da madeira (DIAS, 2000).

A Associação Brasileira de Normas Técnicas (ABNT), em seu documento número 7190 de 1997 (ABNT NBR 7190: 1997) “Projeto de Estruturas de Madeira”, apresenta o Anexo E "Valores médios usuais de resistência e rigidez de algumas madeiras nativas e de reflorestamento”, que fornece os valores médios de algumas propriedades mecânicas e densidade aparente dessas espécies, em que é possível observar que, quanto maior a densidade aparente da madeira de alguma espécie, maiores seus valores de resistência e de rigidez (DIAS; LAHR, 2004; LOBÃO et al., 2004).

Nesse contexto, o objetivo deste trabalho é avaliar, com o uso de modelos de regressão polinomial linear, quadrática e cúbica, a possibilidade da estimativa da tenacidade com o conhecimento da densidade aparente de seis espécies de madeiras nativas (Angico, Jatobá e Paricá) e exóticas (Eucalyptus, Pinus e Teca), abrangendo as classes de resistência fornecidas pela ABNT NBR 7190: 1997.

Revista Árvore, Viçosa-MG, v.38, n.1, p.203-207, 2014
Tabela 1 - Espécies de madeira utilizadas na pesquisa. Table 1 - Wood specimens used in the research.

\begin{tabular}{lcc}
\hline Espécie & $\begin{array}{c}\text { Nome } \\
\text { científico }\end{array}$ & $\begin{array}{c}\text { Classe de } \\
\text { resistência }\end{array}$ \\
\hline Teca & $\begin{array}{c}\text { Tectona grandis } \\
\text { Paricá }\end{array}$ & C 20 \\
Pinus & Schizolobium amazonicum & C 20 \\
Eucalipto & Corys oocarpa Shiede & C 20 \\
Jatobá & Hymenaea sp. & C 40 \\
Angico & Anadenanthera falcata & C 60 \\
\hline
\end{tabular}

\section{MATERIAL E MÉTODOS}

A Tabela 1 apresenta as espécies de madeira utilizadas nesta pesquisa, estando devidamente agrupadas nas classes de resistência da Norma Brasileira ABNT NBR 7190:1997.

Os ensaios de tenacidade (W) foram realizados de acordo com os procedimentos e premissas de cálculo da Norma ASTM D5536-94:1995, fazendo uso do pêndulo de Charpy, e os de densidade aparente $\left(\rho_{12 \%}\right)$ seguiram as recomendações da Norma Brasileira ABNT NBR 7190:1997, ambos executados nas dependências do Laboratório de Madeira e de Estruturas de Madeira (LaMEM) da Escola de Engenharia de São Carlos (EESC/USP). Foram utilizados 18 corpos de prova por espécie de madeira, para realização de cada tipo de ensaio, totalizando 216 corpos de prova.

AAnálise de Variância (ANOVA) foi utilizada para verificar a influência da densidade na obtenção da tenacidade da madeira, avaliada a 5\% de significância, consistindo na equivalência entre médias, como hipótese nula $\left(\mathrm{H}_{0}\right)$ e a não equivalência entre médias como hipótese alternativa $\left(\mathrm{H}_{1}\right)$. P-valor inferior em nível de significância implica rejeitar $\mathrm{H}_{0}$, aceitando-a em caso contrário.

Com o intuito de relacionar a tenacidade das madeiras com a densidade aparente $\left(\mathrm{W} ; \rho_{\mathrm{ap}}\right)$, foram testados modelos de regressão polinomiais do tipo linear, quadrático e cúbico. Para tanto, os modelos de regressão avaliados via análise de variância (ANOVA) foram considerados a 5\% de significância, tendo como hipótese nula a não significância do ajuste fornecido pelo modelo $\left(\beta_{i}=0\right)$ e a da significância $\left(\beta_{i} \neq 0\right)$ do ajuste obtido como hipótese alternativa. $\mathrm{P}$-valor inferior à significância estipulada implica rejeitar $\mathrm{H}_{0}\left(\mathrm{~F}_{\text {dados }}>\mathrm{F}_{\text {crítico }}\right)$, aceitando-a em caso contrário. 


\section{RESULTADOS}

A Tabela 2 apresenta os resultados da densidade aparente e da tenacidade das seis espécies de madeira investigadas, sendo $X_{\mathrm{m}}$ a média amostral, $\mathrm{Cv}$ o coeficiente de variação e Mín e Máx os menores e os maiores valores encontrados.

\section{DISCUSSÕES}

A Tabela 2 indica que os maiores valores de tenacidade da madeira estão associados aos maiores valores da densidade aparente. O P-valor da ANOVA do fator espécie de madeira na obtenção da tenacidade foi igual a 0,000 , revelando ser significativo o tipo de espécie de madeira na obtenção da tenacidade, e os P-valores do teste de Anderson-Darling e de Bartlett e Levene foram, respectivamente, iguais a 0,235; 0,672; e 0,683, revelando ser normal a distribuição dos valores da tenacidade e equivalentes às variâncias entre os tratamentos, validando os resultados da ANOVA.

A Tabela 3 apresenta os resultados do teste de Tukey das espécies de madeira sobre a tenacidade, lembrando-se de que letras iguais implicam tratamentos com médias equivalentes. Os resultados evidenciaram que os maiores valores da tenacidade estão associados às madeiras de maior densidade.

A Tabela 4 e a Figura 1 apresentam e ilustram os resultados dos modelos de regressão da tenacidade em função da densidade aparente.

Os P-valores dos modelos de regressão pela ANOVA foram ambos inferiores ao nível de significância de $5 \%$, implicando ser significativos todos os ajustes obtidos.

Tabela 2 - Resultados da densidade aparente e da tenacidade.

Table 2 - Density and toughness results.

\begin{tabular}{|c|c|c|c|c|c|c|}
\hline \multirow{2}{*}{ Estatística } & \multicolumn{6}{|c|}{$\rho_{\text {ap }}\left(\mathrm{g} / \mathrm{cm}^{3}\right)$} \\
\hline & Paricá & Pinus & Теса & Jatobá & Eucalipto & Angico \\
\hline $\mathrm{X}_{\mathrm{m}}$ & 0,36 & 0,50 & 0,57 & 0,94 & 0,99 & 0,91 \\
\hline Cv (\%) & 19 & 14 & 6 & 12 & 3 & 3 \\
\hline Mín & 0,27 & 0,39 & 0,52 & 0,82 & 0,95 & 0,87 \\
\hline \multirow[t]{2}{*}{ Máx } & 0,45 & 0,64 & 0,63 & 1,08 & 1,09 & 0,97 \\
\hline & \multicolumn{6}{|c|}{ W (J) } \\
\hline Estatística & Paricá & Pinus & Teca & Jatobá & Eucalipto & Angico \\
\hline $\mathrm{X}_{\mathrm{m}}$ & 21,49 & 23,35 & 29,25 & 49,02 & 44,94 & 53,61 \\
\hline $\mathrm{Cv}(\%)$ & 22 & 19 & 12 & 10 & 16 & 8 \\
\hline Mín & 13,51 & 16,05 & 22,87 & 40,82 & 34,33 & 48,96 \\
\hline Máx & 31,17 & 35,06 & 33,74 & 57,59 & 62,56 & 57,50 \\
\hline
\end{tabular}

Tabela 3 - Resultados do teste de Tukey.

Table 3 - Tukey test results.

\begin{tabular}{lcccccc}
\hline Espécie & Paricá & Pinus & Teca & Jatobá & Eucalipto & Angico \\
\hline W $(J)$ & 21,49 & 23,35 & 29,25 & 49,02 & 44,94 & 53,61 \\
Agrupamento & D & D & C & B & B & A \\
\hline
\end{tabular}

Tabela 4 - Modelos de regressão da tenacidade em função da densidade aparente.

Table 4-Regression models of toughness as a function of density.

\begin{tabular}{cccccc}
\hline Tipo de ajuste & Equação & GL & $\mathrm{R}^{2}$ Ajustado (\%) & $\mathrm{F}_{\text {dados }}$ (ANOVA) & P-valor (ANOVA) \\
\hline Linear & $\mathrm{W}=2,41+48,54 \rho_{\mathrm{ap}}$ & 107 & $79,90 \%$ & 426,92 & 0,000 \\
Quadrático & $\mathrm{W}=-8,94+86,28 \rho_{\mathrm{ap}}-27,22 \rho_{\mathrm{ap}^{2}}$ & 107 & $80,60 \%$ & 222,85 & 0,000 \\
Cúbico & $\mathrm{W}=50,76-231,6 \rho_{\mathrm{ap}}+488,8 \rho_{\mathrm{ap}}{ }^{2}-258,5 \rho_{\mathrm{ap}}{ }^{3}$ & 107 & $84,70 \%$ & 198,92 & 0,000 \\
\hline
\end{tabular}




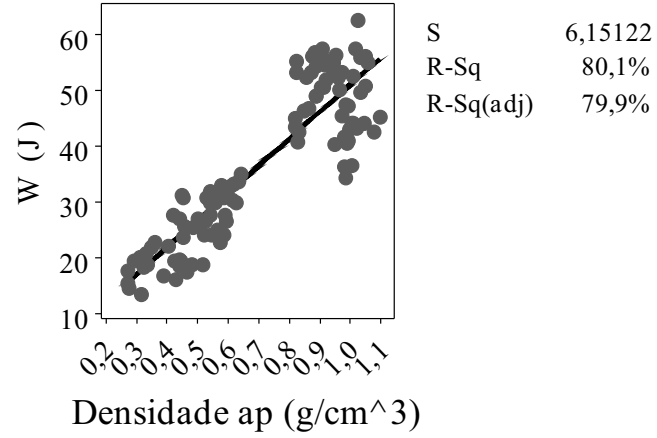

(a)

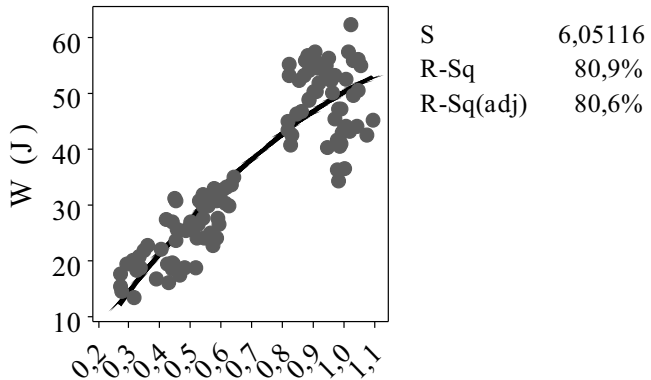

Densidade ap $\left(\mathrm{g} / \mathrm{cm}^{\wedge} 3\right)$

(b)

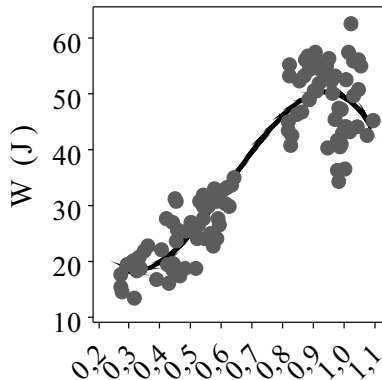

$$
\begin{array}{lr}
\text { S } & 5,36430 \\
\text { R-Sq } & 85,2 \% \\
\text { R-Sq(adj) } & 84,7 \%
\end{array}
$$

Densidade ap $\left(\mathrm{g} / \mathrm{cm}^{\wedge} 3\right)$

(c)

Figura 1 - Gráficos dos modelos de regressão da tenacidade em função da densidade: (a) linear, (b) quadrático e (c) cúbico. Figure 1 - Regression models graphics for toughness as a function of density: (a) linear, (b) quadratic and (c) cubic.

Os valores dos coeficientes de determinação ajustados ( $\mathrm{R}^{2}$ Ajustado) para todas as aproximações foram superiores a 70\%, implicando boa qualidade dos ajustes obtidos (MONTGOMERY, 2005), e o maior valor do coeficiente de determinação $\mathrm{R}^{2}$ Ajustado foi proveniente do modelo cúbico, evidenciando, dessa forma, ser a tenacidade mais bem explicada com o polinômio do terceiro grau.

Adamopoulus e Passialis (2010) também encontraram correlações superiores a 70\% entre tenacidade e módulo de elasticidade na flexão estática em 80 corpos de prova de Picea abies (L.) Karsten, em combinações de anéis de crescimento (anéis finos e espessos) e modo de carregamento (carregamentos nos planos radial e tangencial).

\section{CONCLUSÃO}

Os resultados permitiram a conclusão de que:

- Os modelos de regressão para estimativa da tenacidade mostraram ser significativos em todos os casos, implicando ser possível estimar a tenacidade das madeiras avaliadas com o conhecimento da densidade aparente, por apresentarem coeficientes de determinação ajustados ( $\mathrm{R}^{2}$ Ajustado) superiores a $70 \%$.

- Dos resultados obtidos da análise estatística dos modelos de regressão, o polinomial cúbico forneceu o melhor ajuste das três relações investigadas, seguido dos modelos quadrático e linear.

\section{AGRADECIMENTOS}

Ao Laboratório de Madeiras e de Estruturas de Madeira (LaMEM), do Departamento de Engenharia de Estruturas (SET), da Escola de Engenharia de São Carlos (EESC), da Universidade de São Paulo (USP), pela possibilidade de realização deste trabalho; À Coordenação de Aperfeiçoamento de Pessoal de Nível Superior (CAPES) e ao Conselho Nacional de Desenvolvimento Científico e Tecnológico (CNPq), pelos recursos financeiros concedidos. 


\section{REFERÊNCIAS}

ABRUZZI, R. C. et al. Relação das propriedades mecânicas e densidade de postes de madeira de Eucalipto com seu estado de deterioração, Revista Árvore, v.36, n.6, p. 1173-1181, 2012.

ADAMOPOULUS, S.; PASSIALIS, C. Relationship of toughness and modulus of elasticity in static bending of small clear spruce wood specimens, European Journal of Wood and Wood Products, v.68, p. 109-111, 2010.

ALMEIDA, D. H. et al. Resistência ao impacto na flexão de madeiras nativas e de reflorestamento, Madeira: Arquitetura e Engenharia, v. 12, n. 13, p. 29 - 36, 2011.

\section{AMERICAN SOCIETY FOR TESTING AND} MATERIALS (ASTM). ASTM D5536: Standard methods of testing small clear specimens of timber. Philadelphia; 1995.

ASKELAND, D. R. The science and engineering of materials. 3ed. Boston: PWS Publishing Company, 1994. 876p.

\section{ASSOCIAÇÃO BRASILEIRA DE NORMAS} TÉCNICAS (ABNT). NBR 7190: Projetos de estruturas de madeiras. Rio de Janeiro: 2007.

CALIL JUNIOR, C. et al. Manual de projeto e construção de pontes de madeira. São Carlos: Suprema, 2006. 236p.

CALIL JUNIOR, C.; LAHR, F. A. R.; DIAS, A. A. Dimensionamento de elementos estruturais de madeira. Barueri: Manole, 2003. 152p.

CALLISTER JUNIOR, W. D. Materials science and engineering: an introduction. New York: John Wiley \& Sons, 1999. $600 \mathrm{p}$.

DIAS, F. M.; LAHR, F. A. R. Estimativa de propriedades de resistência e rigidez da madeira através da densidade aparente, Scientia Forestalis, n.65, p. 102-113, 2004.
DIAS, F. M. A densidade aparente como estimador de propriedades de resistência e rigidez da madeira. 2000. 145f. Dissertação (Mestrado em Ciência e Engenharia de Materiais) - Universidade de São Paulo, São Carlos, 2000.

FREITAS, R. R. Modelo teóricoexperimental de deterioração de postes de madeira aplicado ao Estado de São Paulo. 2009. 300f. Tese (Doutorado em Ciência e Engenharia de Materiais) - Universidade de São Paulo, São Carlos, 2009.

HAMM, L. G. et al. Resistência ao impacto da madeira de três espécies florestais. In: ENCONTRO DE PÓS-GRADUAÇÃO, 9., 2007, Pelotas, Rio Grande do Sul. Anais... Pelotas, 2007. (CD-ROM).

LAHR, F. A. R. Considerações a respeito da variabilidade de propriedades de resistência e elasticidade da madeira. 1990. 161f. Tese (Livre Docência), Universidade de São Paulo, São Carlos, 1990.

LOBÃO, M. S. et al. Caracterização das propriedades físico-mecânicas da madeira de Eucalipto com diferentes densidades, Revista Árvore, v. 28, n.6, p. 889-894, 2004.

MONTGOMERY, D. C. Design and analysis of experiments. Arizona: John Wiley \& Sons, 2005, $730 \mathrm{p}$.

MORESCHI, J. C. Propriedades tecnológicas da madeira. Curitiba: UFPR, 2005. 168 p.

RIDOUT, B. Timber decay in buildings: the conservation approach to treatment. London: English Heritage, 2009. 232p.

RITTER, M. Timber bridges: design, construction, inspection and maintenance. Washington: Forest Service, United States Department of Agriculture, 1990. 944 p.

STOLF, D. O. Tenacidade da madeira. 2000. 100f. Dissertação (Mestrado em Ciência e Engenharia de Materiais) - Universidade de São Paulo, São Carlos, 2000.

Revista Árvore, Viçosa-MG, v.38, n.1, p.203-207, 2014 
